\title{
SCALAR PRODUCT FOR THE XXZ SPIN CHAIN WITH GENERAL INTEGRABLE BOUNDARIES
}

\author{
SAMUEL BELLIARD*, RODRIGO A. PIMENTA**, AND NIKITA A. SLAVNOV*** \\ Dedicated to the memory of Omar Foda
}

\begin{abstract}
We calculate the scalar product of Bethe states of the XXZ spin- $\frac{1}{2}$ chain with general integrable boundary conditions. The off-shell equations satisfied by the transfer matrix and the off-shell Bethe vectors allow one to derive a linear system for the scalar product of off-shell and on-shell Bethe states. We show that this linear system can be solved in terms of a compact determinant formula that involves the Jacobian of the transfer matrix eigenvalue and certain q-Pochhammer polynomials of the boundary couplings.
\end{abstract}

Introduction. The anisotropic Heisenberg XXZ spin chain is a paradigmatic model of interacting many body quantum system. It is an integrable model within the quantum inverse scattering method [31, 30. In this paper, we consider the XXZ spin chain on the segment with general integrable boundaries given by the Hamiltonian

$$
H=\epsilon \sigma_{1}^{3}+\kappa^{-} \sigma_{1}^{-}+\kappa^{+} \sigma_{1}^{+}+\sum_{k=1}^{N-1}\left(\sigma_{k}^{x} \otimes \sigma_{k+1}^{x}+\sigma_{k}^{y} \otimes \sigma_{k+1}^{y}+\Delta \sigma_{k}^{3} \otimes \sigma_{k+1}^{3}\right)+\nu \sigma_{N}^{3}+\tau^{-} \sigma_{N}^{-}+\tau^{+} \sigma_{N}^{+},
$$

where $N$ is the length of the chain, $\sigma_{i}^{ \pm, x, y, 3}$ are the standard Pauli matrices acting non-trivially in the $i$-th site of the quantum space $\mathcal{H}=\otimes_{i=1}^{N} \mathbb{C}^{2}$. The anisotropy parameter is $\Delta=\frac{q+q^{-1}}{2}$ with generic $q$. Left $\left\{\epsilon, \kappa^{ \pm}\right\}$and right $\left\{\nu, \tau^{ \pm}\right\}$boundary couplings are also generic. The aim of this paper is to compute the scalar product of on-shell and off-shell Bethe states of the Hamiltonian (1).

The building blocks of the quantum inverse scattering method are the R-matrix, which solves the YangBaxter equations, and the K-matrices, which solve the reflection equation [19, 35. They can be used to construct commuting transfer matrices, which contain the XXZ Hamiltonian with arbitrary boundary couplings (11) as a conserved charge. Solving the spectral problem of the transfer matrix is the primordial aim, followed by the computation of scalar products, form factors and correlation functions (see, e.g., 20]). One of the most powerful methods available to handle such problems is the algebraic Bethe ansatz: it allows the construction of off-shell Bethe states of the transfer matrix as well as certain scalar products in terms of compact formulas (see 32] for a recent review).

Let us recall that the K-matrices are associated with boundary fields at each end of the spin chain. The boundaries in general can break the $U(1)$ symmetry of the bulk Hamiltonian, and it happens when the K-matrices have a general non-diagonal form. This breaking prevents the application of the standard Bethe ansatz technique to analyze the spectral problem of the transfer matrix, unless some constraints on the boundary couplings are imposed (see e.g. [13, 40, 26, 28, 6, 29, 15)1]. In particular, the construction of off-shell states of the transfer matrix in the generic boundary case requires using the modified algebraic Bethe ansatz [5, 7, 16, 8, 1. This method is based on constructing a modified creation operator that satisfies a certain new off-shell relation, which adds an extra term in the transfer matrix eigenvalue. This extra term in the eigenvalue expression is called an inhomogeneous term, firstly discovered 14 within the offdiagonal Bethe ansatz method (ODBA) 37] (see also [27, 23]). We note that on-shell Bethe states have been constructed in the ODBA framework [41. We also recall that study of $U(1)$ breaking boundaries in the XXZ chain has been considered from different and complementary perspectives, including the q-Onsager method [2, 4, the quantum separation of variables [17, 23, 24] and the Q-operator method [25].

Modified algebraic Bethe ansatz was further developed by considering scalar products of Bethe vectors. In particular, considering isotropic XXX spin- $1 / 2$ chain with non-diagonal twist and on the segment with general integrable boundary conditions, compact formulas for the scalar product of on-shell and off-shell

\footnotetext{
${ }^{1}$ We do not intend to give an exhaustive list of references on this previous contributions here, and we refer to paper [7] for more details on the solution of this problem.
} 
Bethe vector have been conjectured [9, 10]. These formulas involve the Jacobian of the transfer matrix eigenvalue, similarly to diagonal boundary cases, but contain a modified factor, which is due to the broken $U(1)$ symmetry. More recently, the case of generic twisted boundary conditions has been proved in 11 , 12 .

It was shown in [12, 33] that the scalar product of on-shell and off-shell Bethe vector satisfies a homogeneous system of linear equations that follow directly from the transfer matrix action on the off-shell Bethe vector. Another key ingredient of the method developed in [12] is the asymptotic behavior of the off-shell Bethe vector when its arguments go to infinity. In this paper, we develop this program for the XXZ chain with general integrable boundary conditions and obtain a compact formula (69), paving the way to further studies of form factors and correlation functions of the model. The formula (69) generalizes the conjecture of 10 to the q-deformed case. It also generalizes previous computations of scalar products [36, 21, 39] which were considered for diagonal or constrained boundary parameters cases.

R-matrix and K-matrices. We recall the basic ingredients of the quantum inverse scattering method. The main object is the R-matrix, given by

$$
R(u)=\frac{1}{q-q^{-1}}\left(\begin{array}{cccc}
q u-q^{-1} u^{-1} & 0 & 0 & 0 \\
0 & u-u^{-1} & q-q^{-1} & 0 \\
0 & q-q^{-1} & u-u^{-1} & 0 \\
0 & 0 & 0 & q u-q^{-1} u^{-1}
\end{array}\right)
$$

The R-matrix acts on the space $\mathbb{C}^{2} \otimes \mathbb{C}^{2}$ and is a solution of the Yang-Baxter equation in $\mathbb{C}^{2} \otimes \mathbb{C}^{2} \otimes \mathbb{C}^{2}$

$$
R_{12}(u / v) R_{13}(u) R_{23}(v)=R_{23}(v) R_{13}(u) R_{12}(u / v) \text {. }
$$

Here the indices $i j$ in $R_{i j}(u)$ indicate the vector spaces where it acts non-trivially. Next, in order to consider the boundary fields, one introduces the reflection matrices

$$
\begin{aligned}
& K^{-}(u)=\left(\begin{array}{cc}
\nu_{-} u+\nu_{+} u^{-1} & \tau^{2}\left(u^{2}-u^{-2}\right) \\
\tilde{\tau}^{2}\left(u^{2}-u^{-2}\right) & \nu_{-} u^{-1}+\nu_{+} u
\end{array}\right), \\
& K^{+}(u)=\left(\begin{array}{cc}
\epsilon_{+} q u+\epsilon_{-} q^{-1} u^{-1} & \tilde{\kappa}^{2}\left(q^{2} u^{2}-q^{-2} u^{-2}\right) \\
\kappa^{2}\left(q^{2} u^{2}-q^{-2} u^{-2}\right) & \epsilon_{-} q u+\epsilon_{+} q^{-1} u^{-1}
\end{array}\right),
\end{aligned}
$$

where $\left\{\nu_{ \pm}, \tau, \tilde{\tau}, \epsilon_{ \pm}, \kappa, \tilde{\kappa}\right\}$ are arbitrary parameters [19, 35]. They are the most general solutions of the reflection equation and of the dual reflection equation $\left[30\right.$, which hold in $\mathbb{C}^{2} \otimes \mathbb{C}^{2}$ :

$$
\begin{aligned}
& R_{12}(u / v) K_{1}^{-}(u) R_{12}(u v) K_{2}^{-}(v)=K_{2}^{-}(v) R_{12}(u v) K_{1}^{-}(u) R_{12}(u / v), \\
& R_{12}(v / u) K_{1}^{+}(u) R_{12}\left(q^{-2} u^{-1} v^{-1}\right) K_{2}^{+}(v)=K_{2}^{+}(v) R_{12}\left(q^{-2} u^{-1} v^{-1}\right) K_{1}^{+}(u) R_{12}(v / u) .
\end{aligned}
$$

From the $R$ and $K^{ \pm}$matrices, one can define the so-called double-row transfer matrix

$$
t(u)=\operatorname{Tr}_{a}\left(K_{a}^{+}(u) T_{a}(u) K_{a}^{-}(u) \hat{T}_{a}(u)\right)
$$

where $a$ denotes an auxiliary $\mathbb{C}^{2}$ vector space, and

$$
\begin{gathered}
T_{a}(u)=R_{a 1}\left(u / x_{1}\right) R_{a 2}\left(u / x_{2}\right) \cdots R_{a N}\left(u / x_{N}\right), \\
\hat{T}_{a}(u)=R_{a N}\left(u x_{N}\right) R_{a N-1}\left(u x_{N-1}\right) \cdots R_{a 1}\left(u x_{1}\right),
\end{gathered}
$$

are called single-row monodromy matrices. They depend on arbitrary parameters $x_{i}$ called inhomogeneities. We denote

$$
K_{a}(u)=T_{a}(u) K_{a}^{-}(u) \hat{T}_{a}(u)=\left(\begin{array}{cc}
\mathscr{A}(u) & \mathscr{B}(u) \\
\mathscr{C}(u) & \mathscr{D}(u)+\frac{q-q^{-1}}{q u^{2}-q^{-1} u^{-2}} \mathscr{A}(u)
\end{array}\right)_{a},
$$

where $\mathscr{A}(u), \mathscr{B}(u), \mathscr{C}(u), \mathscr{D}(u)$ act on $\otimes_{i=1}^{N} \mathbb{C}^{2}$ and are called double-row monodromy operators. It turns out that the transfer matrix (8) enjoys the fundamental property

$$
[t(u), t(v)]=0
$$

for arbitrary $u$ and $v$. Thus, the transfer matrix (8) is a generating function of conserved charges of the model that allows one to reconstruct the Hamiltonian (1) through the relation

$$
H=\left.\frac{q-q^{-1}}{2} \frac{d}{d u} \ln (t(u))\right|_{u=1, w_{i}=1}-\left(N \frac{q+q^{-1}}{2}+\frac{\left(q-q^{-1}\right)^{2}}{2\left(q+q^{-1}\right)}\right) .
$$


In terms of the boundary parameters of the $K^{ \pm}$-matrices, the couplings of the Hamiltonian (11) are expressed as

$$
\begin{array}{lll}
\epsilon=\frac{\left(q-q^{-1}\right)}{2} \frac{\left(\epsilon_{+}-\epsilon_{-}\right)}{\left(\epsilon_{+}+\epsilon_{-}\right)}, & \kappa^{-}=\frac{2\left(q-q^{-1}\right)}{\left(\epsilon_{+}+\epsilon_{-}\right)} \kappa^{2}, & \kappa^{+}=\frac{2\left(q-q^{-1}\right)}{\left(\epsilon_{+}+\epsilon_{-}\right)} \widetilde{\kappa}^{2}, \\
\nu=\frac{\left(q-q^{-1}\right)}{2} \frac{\left(\nu_{-}-\nu_{+}\right)}{\left(\nu_{+}+\nu_{-}\right)}, & \tau^{-}=\frac{2\left(q-q^{-1}\right)}{\left(\nu_{+}+\nu_{-}\right)} \tilde{\tau}^{2}, & \tau^{+}=\frac{2\left(q-q^{-1}\right)}{\left(\nu_{+}+\nu_{-}\right)} \tau^{2} .
\end{array}
$$

For convenience, we will use the following parametrization for the boundary parameters:

$$
\begin{aligned}
& \nu_{-}=i \tilde{\tau} \tau(\mu / \tilde{\mu}+\tilde{\mu} / \mu), \quad \nu_{+}=i \tilde{\tau} \tau(\mu \tilde{\mu}+1 /(\mu \tilde{\mu})), \\
& \epsilon_{-}=i \tilde{\kappa} \kappa(\xi / \tilde{\xi}+\tilde{\xi} / \xi), \quad \epsilon_{+}=i \tilde{\kappa} \kappa(\xi \tilde{\xi}+1 /(\tilde{\xi} \xi)) .
\end{aligned}
$$

The transfer matrix (8) is the object that can be diagonalized by means of the modified algebraic Bethe ansatz [7, 8, 1] which extend the usual boundary algebraic Bethe ansatz [30, 13.

Modified Bethe vectors. Usually, in the Bethe ansatz with $U(1)$ symmetry, the eigenvectors of the transfer matrix are generated using the operators $\mathscr{B}(u)$ or $\mathscr{C}(u)$. In the general case, one needs modified creation and annihilation operators (see [1) given by

$$
\begin{gathered}
\mathscr{B}(u, m)=\frac{q u}{\gamma_{m+1}}\left(\mathscr{B}(u)+\beta q^{m}\left(\frac{q u\left(u^{2}-u^{-2}\right)}{\left(q u^{2}-q^{-1} u^{-2}\right)} \mathscr{A}(u)-u^{-1} \mathscr{D}(u)\right)-\beta^{2} q^{\left.2 m_{\mathscr{C}}(u)\right),}\right. \\
\mathscr{C}(u, m)=-\frac{q u}{\gamma_{m-1}}\left(\mathscr{B}(u)+\alpha q^{-m}\left(\frac{q u\left(u^{2}-u^{-2}\right)}{\left(q u^{2}-q^{-1} u^{-2}\right)} \mathscr{A}(u)-u^{-1} \mathscr{D}(u)\right)-\alpha^{2} q^{\left.-2 m_{\mathscr{C}}(u)\right) .}\right.
\end{gathered}
$$

Here

$$
\gamma_{m}=\alpha q^{-m}-\beta q^{m}, \quad \beta=-i \frac{\tilde{\kappa} \tilde{\xi}}{\kappa \xi} q^{1-2 N}, \quad \alpha=-i \frac{\tilde{\kappa} \xi}{\kappa \tilde{\xi}} q^{1+2 N}
$$

Let

$$
|N\rangle=\otimes_{j=1}^{N}\left(\begin{array}{c}
i q^{N-j} \frac{\mu \tau}{\tilde{\mu} \tilde{\tau} x_{j}} \\
1
\end{array}\right) \quad \text { and } \quad\langle N|=\otimes_{j=1}^{N}\left(\begin{array}{ll}
i q^{N-j} \frac{\mu \tilde{\tau} x_{j}}{\tilde{\mu} \tau} & 1
\end{array}\right) .
$$

Acting on these vectors with the products of the modified creation and annihilation operators we obtain the modified Bethe vectors

$$
|\Psi(\bar{u})\rangle=\mathscr{B}\left(u_{1}, 2(N-1)\right) \mathscr{B}\left(u_{2}, 2(N-2)\right) \cdots \mathscr{B}\left(u_{N}, 0\right)|N\rangle,
$$

and the dual ones

$$
\langle\Psi(\bar{v})|=\langle N| \mathscr{C}\left(v_{1}, 2\right) \mathscr{C}\left(v_{2}, 4\right) \cdots \mathscr{C}\left(v_{N}, 2 N\right) .
$$

We denote $\bar{u}$ and $\bar{v}$ the set of Bethe parameters $\left\{u_{1}, u_{2}, \ldots, u_{N}\right\}$ and $\left\{v_{1}, u_{2}, \ldots, v_{N}\right\}$. When the sets $\bar{u}$ or $\bar{v}$ satisfy the Bethe ansatz equations $\left(\mathcal{Y}\left(u_{i} \mid \bar{u}\right)=0\right.$ or $\mathcal{Y}\left(v_{i} \mid \bar{v}\right)=0$, see (27) below), the vectors (21|22) are called on-shell Bethe vectors. For arbitrary parameters $\bar{u}$ or $\bar{v}$, the vectors (21|22) are called off-shell Bethe vectors.

Off-shell equations of the transfer matrix. Now we are in position to write down the action of the transfer matrix (8) on the off-shell Bethe vectors (2122), following 11. For that, it is convenient to introduce some shorthand notation, namely,

$$
\begin{aligned}
\bar{u}_{i} & =\bar{u} \backslash u_{i}=\left\{u_{1}, \ldots, u_{i-1}, u_{i+1}, \ldots, u_{N}\right\}, \quad \text { for the set with the } u_{i} \text { element removed, } \\
\left\{u, \bar{u}_{i}\right\} & =\left\{u_{1}, \ldots, u_{i-1}, u, u_{i+1}, \ldots, u_{N}\right\}, \quad \text { for the set with the } u_{i} \text { element replaced by } u, \\
Q(u, \bar{u}) & =\prod_{j=1}^{N} Q\left(u, u_{j}\right), \quad \text { for the product of a two-variable function over the set } \bar{u}, \\
Q\left(u, \bar{u}_{i}\right) & =\prod_{j=1, j \neq i}^{N} Q\left(u, u_{j}\right), \quad \text { for the product of a two-variable function over the set } \bar{u}_{i} .
\end{aligned}
$$

If no product is involved we denote, e.g.,

$$
\Lambda(u \mid \bar{u})=\Lambda\left(u \mid u_{1}, \ldots, u_{N}\right),
$$


for multi-variable functions. Using this notation, we have the following compact off-shell relations:

$$
t(u)|\Psi(\bar{u})\rangle=\Lambda(u \mid \bar{u})|\Psi(\bar{u})\rangle+\sum_{i=1}^{N} \frac{F(u)}{F\left(u_{i}\right)} \frac{\mathcal{Y}\left(u_{i} \mid \bar{u}\right)}{Q\left(u_{i},\left\{u, \bar{u}_{i}\right\}\right)}\left|\Psi\left(\left\{u, \bar{u}_{i}\right\}\right)\right\rangle,
$$

and

$$
\langle\Psi(\bar{v})| t(u)=\langle\Psi(\bar{v})| \Lambda(u \mid \bar{v})+\sum_{i=1}^{N} \frac{F(u)}{F\left(v_{i}\right)} \frac{\mathcal{Y}\left(v_{i} \mid \bar{v}\right)}{Q\left(v_{i},\left\{u, \bar{v}_{i}\right\}\right)}\left\langle\Psi\left(\left\{u, \bar{v}_{i}\right\}\right)\right| .
$$

Here

and

$$
\begin{gathered}
F(u)=u^{-1} \frac{q^{2} u^{2}-q^{-2} u^{-2}}{q-q^{-1}}, \quad Q(u, v)=U(u)-U(v), \quad U(u)=\frac{q u^{2}+q^{-1} u^{-2}}{\left(q-q^{-1}\right)^{2}}, \\
\Lambda(u \mid \bar{u})=\phi(u) \frac{Q\left(q^{-1} u, \bar{u}\right)}{Q(u, \bar{u})}+\phi\left(q^{-1} u^{-1}\right) \frac{Q(q u, \bar{u})}{Q(u, \bar{u})}+\frac{H(u)}{Q(u, \bar{u})}
\end{gathered}
$$

The functions $\phi(u)$ and $H(u)$ are given by

$$
\begin{aligned}
& \phi(u)=-\kappa \tilde{\kappa} \tau \tilde{\tau}\left(\tilde{\xi} u+\tilde{\xi}^{-1} u^{-1}\right)\left(\xi^{-1} u+\xi u^{-1}\right)\left(\mu u+\mu^{-1} u^{-1}\right)\left(\tilde{\mu}^{-1} u+\tilde{\mu} u^{-1}\right) \frac{q^{2} u^{2}-q^{-2} u^{-2}}{q u^{2}-q^{-1} u^{-2}} V(u), \\
& H(u)=\left((\kappa \tau)^{2}+(\tilde{\kappa} \tilde{\tau})^{2}+\kappa \tilde{\kappa} \tau \tilde{\tau}\left(\frac{\xi \tilde{\mu}}{\tilde{\xi} \mu} q^{N+1}+\frac{\tilde{\xi} \mu}{\xi \tilde{\mu}} q^{-N-1}\right)\right)\left(u^{2}-u^{-2}\right)\left(q^{2} u^{2}-q^{-2} u^{-2}\right) V(u) V\left(q^{-1} u^{-1}\right),
\end{aligned}
$$

where

$$
V(u)=\prod_{i=1}^{N} Q\left(q^{1 / 2} u, q^{-1 / 2} x_{i}\right)
$$

In the formulas above, $\Lambda(u \mid \bar{u})$ is the eigenvalue of the transfer matrix and $\mathcal{Y}(u \mid \bar{u})$ is called the Bethe function, forming the Baxter TQ equation. We can see that the Bethe function is a linear symmetric function of Bethe parameter $U\left(u_{i}\right)$. The structure of the eigenvalues is similar to the one of the closed XXX case but with this new variable $U(u)$, that is, the Baxter TQ equation can be written solely in terms of $U$. It reflects the crossing invariance of the transfer matrix $t(u)=t\left(q^{-1} u^{-1}\right)$. Therefore, many calculations below are similar to those in 12. The differences appear in the asymptotic calculations which involve the model dependent functions $\phi(u)$ and $H(u)$.

Homogeneous linear system for the scalar product. Now we can follow 12 to fix the general form of the scalar product of on-shell and off-shell Bethe vectors. First of all, we consider the enlarged set $\bar{u}=\left\{u_{1}, \ldots, u_{N+1}\right\}$ and define the following important products:

$$
\begin{aligned}
& F(\bar{u})=\prod_{u_{i} \in \bar{u}} F\left(u_{i}\right), \quad \Delta(\bar{u})=\prod_{\substack{u_{i}, u_{j} \in \bar{u} \\
i<j}} Q\left(u_{i}, u_{j}\right), \quad \Delta^{\prime}(\bar{u})=\prod_{\substack{u_{i}, u_{j} \in \bar{u} \\
i>j}} Q\left(u_{i}, u_{j}\right), \\
& \partial U(u)=\partial_{u} U(u), \quad \partial U(\bar{u})=\prod_{u_{i} \in \bar{u}} \partial U\left(u_{i}\right) .
\end{aligned}
$$

Then we introduce variables $X_{k}=\left\langle\Psi(\bar{v}) \mid \Psi\left(\bar{u}_{k}\right)\right\rangle$, where $\langle\Psi(\bar{v})|$ is an on-shell vector. Let us create an $N+1$ dimensional vector $X=\left(X_{1}, \ldots, X_{N+1}\right)$. It follows from the off-shell equations (23)24) for the transfer matrices and from $\mathcal{Y}\left(v_{i} \mid \bar{v}\right)=0$ with $i=1, \ldots, N$ (since $\langle\Psi(\bar{v})|$ is on-shell) that

$$
L X=0,
$$

where $L$ is the $(N+1) \times(N+1)$ matrix with entries

$$
L_{k j}=\delta_{k j} \Lambda\left(u_{j} \mid \bar{v}\right)-\frac{F\left(u_{k}\right)}{F\left(u_{j}\right)} \frac{\mathcal{Y}\left(u_{j} \mid \bar{u}_{k}\right)}{Q\left(u_{j}, \bar{u}_{j}\right)} .
$$

The homogeneous system (32) has non-trivial solution if $\operatorname{det}_{N+1}(L)=0$, and we now prove that this is indeed the case for (33). Recall that $\operatorname{det}_{N+1}(L)=0$ implies that $\operatorname{rank}(L) \leq N$. 
In order to prove that $\operatorname{det}_{N+1}(L)=0$, let us introduce a nonsingular $(N+1) \times(N+1)$ matrix $W$ with entries

$$
W_{i k}=\frac{Q\left(u_{k}, \bar{w}_{i}\right)}{F\left(u_{k}\right) Q\left(u_{k}, \bar{u}_{k}\right)},
$$

where $\bar{w}=\left\{w_{1}, \ldots, w_{N+1}\right\}$ are generic pairwise distinct parameters. The determinant of $W$ is given by

$$
\operatorname{det}_{N+1}(W)=\frac{1}{F(\bar{u})} \frac{\Delta(\bar{w})}{\Delta(\bar{u})}
$$

The product $\Omega=W L$ has the following entries:

$$
\Omega_{i j}=\frac{1}{F\left(u_{j}\right) Q\left(u_{j}, \bar{u}_{j}\right)}\left(\Lambda\left(u_{j} \mid \bar{v}\right) Q\left(u_{j}, \bar{w}_{i}\right)-\sum_{k=1}^{N+1} \frac{Q\left(u_{k}, \bar{w}_{i}\right)}{Q\left(u_{k}, \bar{u}_{k}\right)} \mathcal{Y}\left(u_{j} \mid \bar{u}_{k}\right)\right) .
$$

To calculate the sum in (36), we use identities

$$
\sum_{k=1}^{N+1} \frac{Q\left(u_{k}, \bar{w}_{i}\right)}{Q\left(u_{k}, \bar{u}_{k}\right)} Q\left(a u_{j}, \bar{u}_{k}\right)=Q\left(a u_{j}, \bar{w}_{i}\right), \quad \sum_{k=1}^{N+1} \frac{Q\left(u_{k}, \bar{w}_{i}\right)}{Q\left(u_{k}, \bar{u}_{k}\right)}=1,
$$

where $a$ is an arbitrary complex number. Setting $a=q$ and $a=q^{-1}$ in (37) and using (27), we obtain

$$
\Omega_{i j}=\frac{1}{F\left(u_{j}\right) Q\left(u_{j}, \bar{u}_{j}\right)}\left(\Lambda\left(u_{j} \mid \bar{v}\right) Q\left(u_{j}, \bar{w}_{i}\right)-\mathcal{Y}\left(u_{j} \mid \bar{w}_{i}\right)\right) .
$$

Setting $\bar{w}_{N+1}=\bar{v}$ we find that the row $\Omega_{N+1, j}$ vanishes. Thus, we have $\operatorname{det}_{N+1}(\Omega)=0$. Therefore, it follows that $\operatorname{det}_{N+1}(W) \operatorname{det}_{N+1}(L)=0$ and $\operatorname{det}_{N+1}(L)=0$.

We now find non-trivial solutions to the system (32). Note that the rows of $\Omega_{i j}$ with $i \leq N$ are given by

$$
\Omega_{i j}=\frac{1}{F\left(u_{j}\right) Q\left(u_{j}, \bar{u}_{j}\right)}\left(\mathcal{Y}\left(u_{j} \mid \bar{v}\right) \frac{Q\left(u_{j}, w\right)}{Q\left(u_{j}, v_{i}\right)}-\mathcal{Y}\left(u_{j} \mid\left\{\bar{v}_{i}, w\right\}\right)\right),
$$

with $w=w_{N+1}$. It is a simple exercise to show that $\Omega_{i j}$ can be rewritten as

$$
\Omega_{i j}=\frac{Q\left(v_{i}, w\right)}{F\left(u_{j}\right) Q\left(u_{j}, \bar{u}_{j}\right) Q\left(u_{j}, v_{i}\right)} \mathcal{Y}\left(u_{j} \mid\left\{\bar{v}_{i}, u_{j}\right\}\right)=\frac{Q\left(v_{i}, w\right)}{\partial U\left(v_{i}\right)} \frac{Q\left(u_{j}, \bar{v}\right)}{F\left(u_{j}\right) Q\left(u_{j}, \bar{u}_{j}\right)} \partial_{v_{i}} \Lambda\left(u_{j} \mid \bar{v}\right) .
$$

Now define $\tilde{\Omega}=V \Omega$ where $V$ is a $(N+1) \times(N+1)$ diagonal matrix with entries $V_{i i}=\frac{\left(1-\delta_{N+1, i}\right)}{Q\left(v_{i}, w\right)}$. We have,

$$
\tilde{\Omega}_{i j}=\frac{M_{i j}}{\partial U\left(v_{i}\right) F\left(u_{j}\right) Q\left(u_{j}, \bar{u}_{j}\right)}, \quad M_{i j}=Q\left(u_{j}, \bar{v}\right) \partial_{v_{i}} \Lambda\left(u_{j} \mid \bar{v}\right)
$$

The system (32) is equivalent to $\tilde{\Omega} X=0$. Let us assume that the rank of $\tilde{\Omega}$ is $N$ and consider the reduced matrix $\tilde{\Omega}_{l}=\tilde{\Omega}_{(N+1),(l)}$ which is obtained from $\tilde{\Omega}$ by removing the row $N+1$ and the column $l$. Then it follows from linear algebra [38] that the system is solved in terms of cofactors

$$
X_{l}=\tilde{G}(\bar{u} \mid \bar{v})(-1)^{N+1+l} \operatorname{det}_{N}\left(\tilde{\Omega}_{l}\right)
$$

where $\tilde{G}(\bar{u} \mid \bar{v})$ is an arbitrary function of $\bar{u}=\left\{u_{1}, \ldots u_{N+1}\right\}$ and $\bar{v}=\left\{v_{1}, \ldots, v_{N}\right\}$. Defining similarly the reduced matrix $M_{l}=M_{(N+1),(l)}$ for the matrix $M$ in (41) with the removed row $N+1$ and column $l$, we have

$$
\begin{aligned}
X_{l} & =\tilde{G}(\bar{u} \mid \bar{v}) \frac{(-1)^{N+1+l} \operatorname{det}_{N}\left(M_{l}\right)}{\partial U(\bar{v}) F\left(\bar{u}_{l}\right) \prod_{j=1, j \neq l}^{N+1} Q\left(u_{j}, \bar{u}_{j}\right)}=\tilde{G}(\bar{u} \mid \bar{v}) \frac{(-1)^{N+1+l} \operatorname{det}_{N}\left(M_{l}\right)}{\partial U(\bar{v}) F\left(\bar{u}_{l}\right) Q\left(\bar{u}_{l}, u_{l}\right) \Delta\left(\bar{u}_{l}\right) \Delta^{\prime}\left(\bar{u}_{l}\right)} \\
& =\frac{(-1)^{N} \tilde{G}(\bar{u} \mid \bar{v})}{\partial U(\bar{v}) \Delta^{\prime}(\bar{u})} \frac{\operatorname{det}_{N}\left(M_{l}\right)}{F\left(\bar{u}_{l}\right) \Delta\left(\bar{u}_{l}\right)} .
\end{aligned}
$$

The equation (43) implies that the ratio

$$
\frac{F\left(\bar{u}_{l}\right) \Delta\left(\bar{u}_{l}\right) X_{l}}{\operatorname{det}_{N}\left(M_{l}\right)}=\frac{F\left(\bar{u}_{j}\right) \Delta\left(\bar{u}_{j}\right) X_{j}}{\operatorname{det}_{N}\left(M_{j}\right)}
$$


for any $l, j \in\{1, \ldots, N+1\}$ is independent of $\bar{u}$. Therefore we can write down the solution as

$$
X_{l}=G(\bar{v}) \frac{\operatorname{det}_{N}\left(M_{l}\right)}{\partial U(\bar{v}) F\left(\bar{u}_{l}\right) \Delta\left(\bar{u}_{l}\right)},
$$

with $G(\bar{v})$ an antisymmetric function of the set $\bar{v}$. Fixing the parameters $\bar{u}$ to a point where we can calculate the "physical" scalar product allows to fix this function. For modified Bethe ansatz models we can calculate the asymptotic behavior of the scalar product for large $\left|u_{j}\right|$ to find it.

Asymptotics of the determinant. For simplicity let us redefine $\bar{u}=\bar{u}_{N+1}$. To take the limit $u_{j} \rightarrow \infty$, $j=1, \ldots, N$, we have to perform again a transformation of the determinant in (45). First, using (40) we can rewrite it as

$$
\operatorname{det}_{N}\left(M_{N+1}\right)=\operatorname{det}_{N}\left(Q\left(u_{j}, \bar{v}\right) \partial_{v_{i}} \Lambda\left(u_{j} \mid \bar{v}\right)\right)=\partial U(\bar{v}) \operatorname{det}_{N}\left(\frac{\mathcal{Y}\left(u_{j} \mid\left\{\bar{v}_{i}, u_{j}\right\}\right)}{Q\left(u_{j}, v_{i}\right)}\right) .
$$

Let us consider $N \times N$ matrix $B$ with entries and determinant given by

$$
B_{k l}=\frac{Q\left(\bar{u}, v_{l}\right)}{Q\left(u_{k}, v_{l}\right) Q\left(\bar{v}_{l}, v_{l}\right)}, \quad \operatorname{det}_{N}(B)=\frac{\Delta^{\prime}(\bar{u})}{\Delta^{\prime}(\bar{v})} .
$$

Using the matrix $B$ we can rewrite the determinant (46) as

$$
\operatorname{det}_{N}\left(M_{N+1}\right)=\frac{\operatorname{det}_{N}\left(B M_{N+1}\right)}{\operatorname{Det}_{N}(B)}=\partial U(\bar{v}) \frac{\Delta^{\prime}(\bar{v})}{\Delta^{\prime}(\bar{u})} \operatorname{det}_{N}\left(\sum_{l=1}^{N} \frac{\mathcal{Y}\left(u_{j} \mid\left\{\bar{v}_{l}, u_{j}\right\}\right)}{Q\left(u_{j}, v_{l}\right) Q\left(u_{k}, v_{l}\right)} \frac{Q\left(\bar{u}, v_{l}\right)}{Q\left(\bar{v}_{l}, v_{l}\right)}\right) .
$$

The summation in (48) is completely analogous to the XXX case considered in 12. It is based on the fact that the Bethe function (27) is a symmetric polynomial of the first degree in the variables $U\left(u_{i}\right)$. One has to consider the chain rule $\partial_{U}=\frac{\partial_{u}}{\partial U(u)}$ to find that

$$
\operatorname{det}_{N}\left(M_{N+1}\right)=\partial U(\bar{v}) \Delta^{\prime}(\bar{v}) \Delta(\bar{u}) \operatorname{det}_{N}\left(\delta_{i j} \Lambda\left(u_{i} \mid \bar{v}\right)+\frac{\left.\partial_{u_{j}} \mathcal{Y}(w \mid \bar{u})\right|_{w=u_{i}}}{Q\left(u_{i}, \bar{u}_{i}\right) \partial U\left(u_{j}\right)}\right) .
$$

Therefore,

$$
\frac{\operatorname{det}_{N}\left(M_{N+1}\right)}{\partial U(\bar{v}) \Delta(\bar{u}) F(\bar{u})}=\frac{\Delta^{\prime}(\bar{v})}{F(\bar{u})} \operatorname{det}_{N}\left(\delta_{i j} \Lambda\left(u_{i} \mid \bar{v}\right)+\frac{\left.\partial_{u_{j}} \mathcal{Y}(w \mid \bar{u})\right|_{w=u_{i}}}{Q\left(u_{i}, \bar{u}_{i}\right) \partial U\left(u_{j}\right)}\right) .
$$

Now the limit of the determinant at $u_{i} \rightarrow \infty$ can be easily calculated. To do this, we consider the asymptotic behavior of the matrix elements. First we put $u_{i}=u a^{i}$ for $i=1, \ldots, N$ in (26), with $a$ some generic parameter and then consider the leading term in $u$. A straightforward calculation gives

$$
\Lambda\left(u_{i}, \bar{v}\right)=\frac{\left(q\left(u a^{i}\right)^{2}\right)^{N+2}}{\left(q-q^{-1}\right)^{2 N}}\left(\tilde{\kappa}^{2} \tilde{\tau}^{2}+\kappa^{2} \tau^{2}\right)+\cdots,
$$

and

$$
\frac{\left.\partial_{u_{j}} \mathcal{Y}(w \mid \bar{u})\right|_{w=u_{i}}}{Q\left(u_{i}, \bar{u}_{i}\right) \partial U\left(u_{j}\right)}=\frac{\left(q\left(u a^{i}\right)^{2}\right)^{N+2}}{\left(q-q^{-1}\right)^{2 N}} \kappa \tilde{\kappa} \tau \tilde{\tau}\left(\frac{\mu \tilde{\xi}}{\tilde{\mu} \xi} \Xi_{i j}^{q^{-1}}+\frac{\tilde{\mu} \xi}{\mu \tilde{\xi}} \Xi_{i j}^{q}\right)+\cdots,
$$

with

$$
\Xi_{i j}^{q}=\frac{\prod_{k=1, k \neq j}^{N}\left(q a^{2 i}-q^{-1} a^{2 k}\right)}{\prod_{k=1, k \neq i}^{N}\left(a^{2 i}-a^{2 k}\right)} .
$$

Here and further on, the ellipsis denotes subleading terms compared to the leading asymptotics at $u \rightarrow \infty$.

Using (5152) we obtain the following asymptotic behavior of the determinant:

$$
\frac{\operatorname{det}_{N}\left(M_{N+1}\right)}{\partial U(\bar{v}) \Delta(\bar{u}) F(\bar{u})}=\frac{q^{N^{2}}\left(u^{N} a^{\frac{N(N+1)}{2}}\right)^{2 N+3} \Delta^{\prime}(\bar{v})}{\left(q-q^{-1}\right)^{2 N^{2}-N}} \nu_{N}+\cdots,
$$


with

$$
\begin{aligned}
\nu_{N} & =\operatorname{det}_{N}\left(\left(\tilde{\kappa}^{2} \tilde{\tau}^{2}+\kappa^{2} \tau^{2}\right) \delta_{i j}+\kappa \tilde{\kappa} \tau \tilde{\tau}\left(\frac{\mu \tilde{\xi}}{\tilde{\mu} \xi} \Xi_{i j}^{q^{-1}}+\frac{\tilde{\mu} \xi}{\mu \tilde{\xi}} \Xi_{i j}^{q}\right)\right) \\
& =\left(\kappa^{2} \tau^{2}\right)^{N}\left(-\frac{\tilde{\kappa} \tilde{\tau} \tilde{\mu} \xi}{\kappa \tau \mu \tilde{\xi}} q^{1-N} ; q^{2}\right)_{N}\left(-\frac{\tilde{\kappa} \tilde{\tau} \mu \tilde{\xi}}{\kappa \tau \tilde{\mu} \xi} q^{1-N} ; q^{2}\right)_{N} .
\end{aligned}
$$

Here

$$
(b ; q)_{n}=\prod_{k=0}^{n-1}\left(1-b q^{k}\right)
$$

is the q-Pochhammer symbol.

Asymptotics of the Bethe vectors. The next step is to compute the asymptotic behavior of the scalar product $\langle\Psi(\bar{v}) \mid \Psi(\bar{u})\rangle$ through the objects of the quantum inverse scattering method. Using the R-matrix (2) we obtain for $u \rightarrow \infty$

$$
\left(\prod_{i=1}^{N} x_{i}\right) T_{a}(u)=\left(\frac{q u^{2}}{\left(q-q^{-1}\right)^{2}}\right)^{N / 2}\left(\begin{array}{cc}
q^{S^{3}} & 0 \\
0 & q^{-S^{3}}
\end{array}\right)+\left(\frac{q u^{2}}{\left(q-q^{-1}\right)^{2}}\right)^{(N-1) / 2}\left(\begin{array}{cc}
0 & S^{-} \\
S^{+} & 0
\end{array}\right)+\cdots
$$

and

$$
\left(\prod_{i=1}^{N} x_{i}\right)^{-1} \hat{T}_{a}(u)=\left(\frac{q u^{2}}{\left(q-q^{-1}\right)^{2}}\right)^{N / 2}\left(\begin{array}{cc}
q^{S^{3}} & 0 \\
0 & q^{-S^{3}}
\end{array}\right)+\left(\frac{q u^{2}}{\left(q-q^{-1}\right)^{2}}\right)^{(N-1) / 2}\left(\begin{array}{cc}
0 & \hat{S}^{-} \\
\hat{S}^{+} & 0
\end{array}\right)+\cdots
$$

The $U_{q}\left(s l_{2}\right)$ operators in (57/58) are

$$
S^{3}=\sum_{i=1}^{N} \frac{\sigma_{i}^{3}}{2}, \quad S^{ \pm}=\sum_{i=1}^{N} x_{i} q^{\mp \sum_{j=1}^{i-1} \frac{\sigma_{j}^{3}}{2}} \sigma_{i}^{ \pm} q^{ \pm \sum_{j=i+1}^{N} \frac{\sigma_{j}^{3}}{2}},
$$

and

$$
\hat{S}^{ \pm}=\sum_{i=1}^{N} x_{i}^{-1} q^{ \pm \sum_{j=1}^{i-1} \frac{\sigma_{j}^{3}}{2}} \sigma_{i}^{ \pm} q^{\mp \sum_{j=i+1}^{N} \frac{\sigma_{j}^{3}}{2}}
$$

Substituting (57/58) in (11) we obtain for $u \rightarrow \infty$

$$
K_{a}(u)=\left(\frac{q u^{2}}{\left(q-q^{-1}\right)^{2}}\right)^{N}\left(\begin{array}{cc}
A u & \tau^{2} u^{2} \\
\tilde{\tau}^{2} u^{2} & A^{*} u
\end{array}\right)+\cdots
$$

where

$$
\begin{aligned}
A & =\nu_{-} q^{2 S^{3}}+\left(q-q^{-1}\right) q^{-1 / 2}\left(\tilde{\tau}^{2} S^{-} q^{S^{3}}+\tau^{2} q^{S^{3}} \hat{S}^{+}\right), \\
A^{*} & =\nu_{+} q^{-2 S^{3}}+\left(q-q^{-1}\right) q^{-1 / 2}\left(\tau^{2} S^{+} q^{-S^{3}}+\tilde{\tau}^{2} q^{-S^{3}} \hat{S}^{-}\right),
\end{aligned}
$$

that satisfy the q-deformed Dolan-Grady relations 2],

$$
\begin{aligned}
{\left[A,\left[A,\left[A, A^{*}\right]_{q}\right]_{q^{-1}}\right] } & =\left(q^{2}-q^{-2}\right)^{2} \tau^{2} \tilde{\tau}^{2}\left[A, A^{*}\right], \\
{\left[A^{*},\left[A^{*},\left[A^{*}, A\right]_{q}\right]_{q^{-1}}\right] } & =\left(q^{2}-q^{-2}\right)^{2} \tau^{2} \tilde{\tau}^{2}\left[A^{*}, A\right],
\end{aligned}
$$

where $[A, B]_{q}=q A B-q^{-1} B A$.

It follows from (61) that the modified operator (17) behaves as

$$
\mathscr{B}(u, m)=\left(\frac{q u^{2}}{\left(q-q^{-1}\right)^{2}}\right)^{N} q u^{3} \frac{\beta q^{m}}{\gamma_{m+1}}\left(A+q^{-m} \beta^{-1} \tau^{2}-q^{m} \beta \tilde{\tau}^{2}\right)+\cdots,
$$

as $u \rightarrow \infty$.

The action of $A$ on the vector (20) follows from the representation theory of the q-Onsager algebra [3]. In our notation, the action of $A$ is

$$
A|N\rangle=i \tau \tilde{\tau}\left(q^{N} \frac{\mu}{\tilde{\mu}}+q^{-N} \frac{\tilde{\mu}}{\mu}\right)|N\rangle .
$$


Therefore,

$$
\mathscr{B}(u, m)|N\rangle=\left(\frac{q u^{2}}{\left(q-q^{-1}\right)^{2}}\right)^{N} q u^{3} \tau^{2} \frac{\left(1+i \frac{\tilde{\mu} \tilde{\tau}}{\mu \tau} q^{-N+m} \beta\right)\left(1+i \frac{\mu \tilde{\tau}}{\tilde{\mu} \tau} q^{N+m} \beta\right)}{\gamma_{m+1}}|N\rangle+\cdots,
$$

as $u \rightarrow \infty$. Formulas (67) and (21) imply that the scalar product $\langle\Psi(\bar{v}) \mid \Psi(\bar{u})\rangle$ has the following asymptotics:

$$
\langle\Psi(\bar{v}) \mid \Psi(\bar{u})\rangle=\frac{(\bar{u})^{2 N+3}\langle\Psi(\bar{v}) \mid N\rangle}{\left(q-q^{-1}\right)^{2 N^{2}}}\left(i \frac{\kappa \tilde{\xi}}{\tilde{\kappa} \xi} \tau^{2}\right)^{N} \frac{\left(-\frac{\tilde{\kappa} \tilde{\tau} \tilde{\xi} \tilde{\mu}}{\kappa \tau \xi \mu} q^{1-3 N} ; q^{2}\right)_{N}\left(-\frac{\tilde{\kappa} \tilde{\tau} \mu \tilde{\xi}}{\kappa \tau \xi \tilde{\mu}} q^{1-N} ; q^{2}\right)_{N}}{\left(\frac{\tilde{\xi}^{2}}{\xi^{2}} q^{2-4 N} ; q^{4}\right)_{N}}+\cdots,
$$

for $u_{i} \rightarrow \infty$.

Determinant formula. We are in position to present the scalar product of the on-shell dual Bethe vectors $\langle\Psi(\bar{v})|$ and off-shell Bethe vectors $|\Psi(\bar{u})\rangle$. To do this, it is enough to compare (68) with the formula (45) for $u_{i} \rightarrow \infty$ (see (54)). This allows us to find the unknown function $G(\bar{v})$. A simple calculation shows that

$$
\langle\Psi(\bar{v}) \mid \Psi(\bar{u})\rangle=\eta\langle\Psi(\bar{v}) \mid N\rangle \frac{\operatorname{det}_{N}\left(Q\left(u_{j}, \bar{v}\right) \partial_{v_{i}} \Lambda\left(u_{j} \mid \bar{v}\right)\right)}{\partial U(\bar{v}) \Delta^{\prime}(\bar{v}) F(\bar{u}) \Delta(\bar{u})},
$$

where

$$
\eta=\left(\frac{i \tilde{\xi}}{q^{N}\left(q-q^{-1}\right) \tilde{\kappa} \kappa \xi}\right)^{N} \frac{\left(-\frac{\tilde{\kappa} \tilde{\tau} \tilde{\mu} \tilde{\xi}}{\kappa \tau \mu \xi} q^{1-3 N} ; q^{2}\right)_{N}}{\left(\frac{\tilde{\xi}^{2}}{\xi^{2}} q^{2-4 N} ; q^{4}\right)_{N}\left(-\frac{\tilde{\kappa} \tilde{\mu} \tilde{\xi}}{\kappa \tau \mu \xi} q^{1-N} ; q^{2}\right)_{N}} .
$$

This is the main result of the paper. Recall that the functions entering (69) are given in (25]26]27]31).

XXX limit. We can now check the conjecture of [10] for the XXX chain. We need to consider the change of variables

$$
\begin{aligned}
& u=e^{\hbar \lambda}, \quad v=e^{\hbar v}, \quad x=e^{\hbar \theta}, \quad q=e^{\hbar}, \quad \epsilon_{ \pm}= \pm \frac{e^{ \pm \hbar \mathfrak{q}}}{e^{\hbar}-e^{-\hbar}}, \quad \nu_{ \pm}=\mp \frac{e^{\mp \hbar p}}{e^{\hbar}-e^{-\hbar}}, \quad \frac{\tilde{\xi}}{\xi}=i \frac{\rho}{\sqrt{\xi^{+} \xi^{-}}} \\
& \kappa^{2}=\frac{\xi^{-}}{2\left(e^{\hbar}-e^{-\hbar}\right)}, \quad \tilde{\kappa}^{2}=\frac{\xi^{+}}{2\left(e^{\hbar}-e^{-\hbar}\right)}, \quad \tau^{2}=\frac{\eta^{+}}{2\left(e^{\hbar}-e^{-\hbar}\right)}, \quad \tilde{\tau}^{2}=\frac{\eta^{-}}{2\left(e^{\hbar}-e^{-\hbar}\right)}, \quad \frac{\mu}{\tilde{\mu}}=i \frac{\sqrt{\eta^{+} \eta^{-}}}{\tilde{\rho}},
\end{aligned}
$$

and take the limit $\hbar \rightarrow 0$. We find for the limit of the eigenvalue

$$
\Lambda_{X}(\lambda \mid \bar{\lambda})=\phi_{X}(\lambda) \frac{Q_{X}(\lambda-1, \bar{\lambda})}{Q_{X}(\lambda, \bar{\lambda})}+\phi_{X}(-\lambda-1) \frac{Q_{X}(\lambda+1, \bar{\lambda})}{Q_{X}(\lambda, \bar{\lambda})}+\frac{H_{X}(\lambda)}{Q_{X}(\lambda, \bar{\lambda})},
$$

where

$$
\begin{aligned}
Q_{X}(\lambda, \theta) & =U_{X}(\lambda)-U_{X}(\theta), \quad U_{X}(\lambda)=\lambda(\lambda+1), \quad \partial U_{X}(\lambda)=2 \lambda+1, \\
\phi_{X}(\lambda) & =(\mathfrak{q}+\lambda(1-\rho))(p+\lambda(1-\tilde{\rho})) V_{X}(\lambda) \frac{2 \lambda+2}{2 \lambda+1} \\
H_{X}(u) & =\left(\xi^{-} \eta^{+}+\xi^{+} \eta^{-}+2-2(\rho-1)(\tilde{\rho}-1)\right) \lambda(\lambda+1) V_{X}(\lambda) V_{X}(-\lambda-1), \\
V_{X}(\lambda) & =\prod_{i=1}^{N} Q\left(\lambda+1 / 2, \theta_{i}-1 / 2\right), \quad F_{X}(\lambda)=2 \lambda+2 .
\end{aligned}
$$

Here $(1-\tilde{\rho})^{2}=1+\eta^{+} \eta^{-}$and $(1-\rho)^{2}=1+\xi^{+} \xi^{-}$.

Then we obtain for the scalar product

$$
\left\langle\Psi_{X}(\bar{v}) \mid \Psi_{X}(\bar{\lambda})\right\rangle=\left(\frac{(\rho-2)}{2(\rho-1)^{2}} \frac{\eta^{+} \eta^{-}}{\tilde{\rho}^{2}} \frac{1+\frac{\tilde{\rho} \rho}{\eta^{+} \xi^{-}}}{1-\frac{\tilde{\rho} \xi^{+}}{\eta^{+} \rho}}\right)^{N}\langle X|\tilde{\mathscr{C}}(\bar{v})| X\rangle \frac{\operatorname{det}_{N}\left(Q_{X}\left(\lambda_{j}, \bar{v}\right) \partial_{v_{i}} \Lambda_{X}\left(\lambda_{j} \mid \bar{v}\right)\right)}{\partial U_{X}(\bar{v}) \Delta_{X}^{\prime}(\bar{v}) F_{X}(\bar{\lambda}) \Delta_{X}(\bar{\lambda})}
$$


where we used $\xi^{+} \xi^{-}=\rho(\rho-2)$. The operator $\tilde{\mathscr{C}}(v)$ and the vectors $|X\rangle$ and $\langle X|$ are given by

$$
\begin{aligned}
& \tilde{\mathscr{C}}(v)=\frac{\xi^{+}}{\rho} \mathscr{C}_{X}(v)+\left(\frac{2 v}{2 v+1} \mathscr{A}_{X}(v)-\mathscr{D}_{X}(v)\right)-\frac{\rho}{\xi^{+}} \mathscr{B}_{X}(v), \\
& |X\rangle=\otimes_{j=1}^{N}\left(\begin{array}{c}
1 \\
-\frac{\tilde{\rho}}{\eta^{+}}
\end{array}\right), \quad\langle X|=\otimes_{j=1}^{N}\left(\begin{array}{ll}
1 & -\frac{\tilde{\rho}}{\eta^{-}}
\end{array}\right) .
\end{aligned}
$$

Taking the limit $\tilde{\rho} \rightarrow 0$ and then $\eta^{ \pm} \rightarrow 0$ of the ratio $\left\langle\Psi_{X}(\bar{v}) \mid \Psi_{X}(\bar{\lambda})\right\rangle /\left(\frac{\eta^{+} \eta^{-}}{\tilde{\rho}^{2}}\right)^{N}$ we recover the conjecture of [10] and thus prove it.

Conclusion. In this paper, we computed the scalar product of on-shell and off-shell modified Bethe vectors associated with the XXZ spin chain with general integrable boundary conditions. Similarly to the $U(1)$ invariant models, the compact formula is given by a determinant involving the Jacobian of the transfer matrix eigenvalue. Breaking the $U(1)$ symmetry implies the expectation value $\langle\Psi(\bar{v}) \mid N\rangle$, while the "dynamic" nature of the modified creation operators generates q-Pochhammer symbols involving the boundary parameters fields. The first point to be investigated from here is the computation of the expectation value $\langle\Psi(\bar{v}) \mid N\rangle$ and check whether it can be written as a boundary case of the q-deformed modified Izergin determinant [11, 34, 18. The next natural step is to compute the action of local operators on the modified Bethe vector, and then derive building blocks of correlation functions, trying to generalize the known results for the diagonal boundary case 21, 22. Certainly, the solution of these problems will bring advances to the solution of the inverse problem for models without $U(1)$ symmetry, providing new tools for the calculation of physical quantities.

Acknowledgments. R.P. thanks the Institute Denis Poisson, where a part of this work was done, for hospitality, and Pascal Baseilhac for discussions and encouragement. R.P. was supported by CNPq (grant \# 150829/2020-5). N.S. thanks the CNRS for his grant to join the Institute Denis Poisson of the University of Tours where a part of this work was done.

\section{REFERENCES}

[1] J. Avan, S. Belliard, N. Grosjean and R.A. Pimenta, Modified algebraic Bethe ansatz for XXZ chain on the segment - III - Proof, , Nucl. Phys. B 899 (2015) 229, arXiv:1506.02147

[2] P. Baseilhac, An integrable structure related with tridiagonal algebras, Nucl. Phys. B 705 (2005) 605-619, arXiv: math-ph/0408025

[3] P. Baseilhac, A family of tridiagonal pairs and related symmetric functions, J. Phys. A: Math. Gen. 39 (2006) 11773 , arXiv:math-ph/0604035

[4] P. Baseilhac and K. Koizumi, Exact spectrum of the XXZ open spin chain from the q-Onsager algebra representation theory, J. Stat. Mech. (2007) P09006, arXiv:hep-th/0703106

[5] S. Belliard and N. Crampé, Heisenberg XXX Model with General Boundaries: Eigenvectors from Algebraic Bethe Ansatz, SIGMA 9 (2013), 072, arXiv: 1309.6165.

[6] S. Belliard, N. Crampé and E. Ragoucy, Algebraic Bethe ansatz for open XXX model with triangular boundary matrices, Lett. Math. Phys. 103 (2013) 493, arXiv:1209.4269

[7] S. Belliard, Modified algebraic Bethe ansatz for XXZ chain on the segment - I: Triangular cases, Nucl. Phys. B 892 (2015) 1, arXiv:1408.4840

[8] S. Belliard and R.A. Pimenta, Modified algebraic Bethe ansatz for XXZ chain on the segment - II - general cases, Nucl. Phys. B 894 (2015) 527, arXiv:1412.7511.

[9] S. Belliard and R.A. Pimenta, Slavnov and Gaudin-Korepin Formulas for Models without U(1) Symmetry: the Twisted XXX Chain, SIGMA 11 (2015) 099, arXiv:1506.06550

[10] S. Belliard and R.A. Pimenta, Slavnov and Gaudin-Korepin Formulas for Models without U(1) Symmetry: the XXX chain on the segment, J. Phys. A: Math. Theor. 49 (2016) 17LT01, arXiv:1507.03242

[11] S. Belliard and N.A. Slavnov, Scalar products in twisted XXX spin chain. Determinant representation, SIGMA 15 (2019) 066, arXiv:1906.06897 [math-ph]

[12] S. Belliard and N.A. Slavnov, Why scalar products in the algebraic Bethe ansatz have determinant representation, JHEP 10 (2019) 103 arXiv:1908.00032 [math-ph].

[13] J. Cao, H.-Q. Lin, K. Shi and Y. Wang, Exact solution of XXZ spin chain with unparallel boundary fields, Nucl. Phys. B663 (2003) 487

[14] J. Cao, W. Yang, K. Shi and Y. Wang, Off-diagonal Bethe ansatz and exact solution of a topological spin ring, Phys. Rev. Lett. 111 (2013) 137201, arXiv:1305.7328 
[15] N. Cirilo António, N. Manojlović and I. Salom, Algebraic Bethe ansatz for the XXX chain with triangular boundaries and Gaudin model, Nucl. Phys. B 889 (2014) 87, arXiv:1405.7398

[16] N. Crampé, Algebraic Bethe ansatz for the totally asymmetric simple exclusion process with boundaries, J. Phys. A: Math. Theor. 48 (2015) 08FT01, arXiv: 1411.7954

[17] S. Faldella, N. Kitanine and G. Niccoli, Complete spectrum and scalar products for open spin-1/2 XXZ quantum chains with non-diagonal boundary terms, J. Stat. Mech. (2014) P01011, arXiv:1307.3960

[18] O. Foda and M. Wheeler, Partial domain wall partition functions, J. High Energ. Phys. 7 (2012) 186, arXiv:1205.4400

[19] S. Ghoshal and A.B. Zamolodchikov, Boundary S-matrix and boundary state in two-dimensional integrable quantum field theory, Int. J. Mod. Phys. A 9 (1994) 3841, arXiv:hep-th/9306002

[20] V.E. Korepin, N.M. Bogoliubov and A.G. Izergin, Quantum Inverse Scattering Method and Correlation Functions, Cambridge Univ. Press (1993).

[21] N. Kitanine, K.K. Kozlowski, J.M. Maillet, G. Niccoli, N.A. Slavnov and V. Terras, Correlation functions of the open XXZ chain I, J. Stat. Mech. (2007) P10009, arXiv:0707.1995

[22] N. Kitanine, K.K. Kozlowski, J.M. Maillet, G. Niccoli, N.A. Slavnov and V. Terras, Correlation functions of the open XXZ chain II, J. Stat. Mech. (2008) P07010, arXiv:0803.3305

[23] N. Kitanine, J.M. Maillet and G. Niccoli, Open spin chains with generic integrable boundaries: Baxter equation and Bethe ansatz completeness from separation of variables, J. Stat. Mech. (2014) P05015, arXiv:1401.4901

[24] N. Kitanine, J. M. Maillet, G. Niccoli and V. Terras, The open XXZ spin chain in the SoV framework: scalar product of separate states, J. Phys. A: Math. Theor. 51 (2018) 485201, arXiv:1807.05197 [math-ph]

[25] A. Lazarescu and V. Pasquier, Bethe Ansatz and Q-operator for the open ASEP J. Phys. A: Math. Theor. 47 (2014) 295202, arXiv: 1403.6963

[26] R.I. Nepomechie, Bethe Ansatz solution of the open XXZ chain with nondiagonal boundary terms, J. Phys. A37 (2004) 433, arXiv:hep-th/0304092

[27] R.I. Nepomechie, An inhomogeneous T-Q equation for the open XXX chain with general boundary terms: completeness and arbitrary spin, J. Phys. A: Math. Theor. 46442002

[28] R.I. Nepomechie and F. Ravanini, Completeness of the Bethe Ansatz solution of the open XXZ chain with nondiagonal boundary terms, J. Phys. A36 (2003), Addendum ibid A 37 (2004) 1945, arXiv:hep-th/0307095

[29] R.A. Pimenta and A. Lima-Santos, Algebraic Bethe ansatz for the six vertex model with upper triangular $K$-matrices, J. Phys. A: Math. Theor. 46 (2013) 455002, arXiv:1308.4446

[30] E.K. Sklyanin, Boundary conditions for integrable quantum systems, J. Phys. A21 (1988) 2375.

[31] E.K. Sklyanin, L.A. Takhtadzhyan and L.D. Faddeev, The Quantum Inverse Problem Method. I, Theor. Math. Phys. 40 (1979), 688

[32] N. Slavnov, Algebraic Bethe ansatz, (2018) arXiv:1804.07350.

[33] N. Slavnov, A. Zabrodin, A. Zotov, Scalar products of Bethe vectors in the 8-vertex model, JHEP 06 (2020) 123, arXiv:2005.11224

[34] O. Tsuchiya, Determinant formula for the six-vertex model with reflecting end, J. Math. Phys. 39 (1998), 5946, arXiv: solv-int/9804010.

[35] H. de Vega and A. Gonzalez-Ruiz, "Boundary K matrices for the XYZ, XXZ and XXX spin chains," J. Phys. A 27 (1994), 6129-6138 arXiv:hep-th/9306089 [hep-th]

[36] Y.-S. Wang, The scalar products and the norm of Bethe eigenstates for the boundary XXX Heisenberg spin-1/2 finite chain, Nucl.Phys. B 622 (2002) 633

[37] Y. Wang, W.-L. Yang, J. Cao and K. Shi, Off-Diagonal Bethe Ansatz for Exactly Solvable Models, Springer-Verlag Berlin Heidelberg, (2015).

[38] P.A. White, Linear algebra, Dichenson Publishing Co., Inc. Belmont (1966)

[39] W. L. Yang, X. Chen, J. Feng, K. Hao, B. Y. Hou, K. J. Shi and Y. Z. Zhang, Determinant representations of scalar products for the open XXZ chain with non-diagonal boundary terms, JHEP 1101 (2011) 006 arXiv:1011.4719

[40] W.-L. Yang and Y.-Z. Zhang, On the second reference state and complete eigenstates of the open XXZ chain, JHEP 0704 (2007) 044, arXiv:hep-th/0703222

[41] X. Zhang, Y.Y. Li, J. Cao, W.-L. Yang, K. Shi, Y. Wang, Bethe states of the XXZ spin-1/2 chain with arbitrary boundary fields, Nucl. Phys. B893 (2015) 70, arXiv:1412.6905

* Institut Denis-Poisson CNRS/UMR 7013 - Université de Tours - Université D’Orléans Parc de Grammont, 37200 TOuRs, FRANCE

Email address: samuel.belliard@gmail.com

***Departamento de Física, Universidade Federal de lavras, Caixa Postal 3037, 37200-000, Lavras, MG, BRaZiL

Instituto de Física de São Carlos, Universidade de São Paulo, Caixa Postal 369, 13.560-590, São Carlos, SP, BRAZIL

Email address: pimenta@ifsc.usp.br

Steklov Mathematical Institute of Russian Academy of Sciences, 8 Gubkina str., Moscow, 119991, Russia

Email address: nslavnov@mi-ras.ru 